Published in final edited form as:

Phys Med Biol. 2012 November 7; 57(21): 6807-6826. doi:10.1088/0031-9155/57/21/6807.

\title{
CT to Cone-beam CT Deformable Registration With Simultaneous Intensity Correction
}

\author{
Xin Zhen ${ }^{1,2}$, Xuejun Gu ${ }^{1}$, Hao Yan ${ }^{1}$, Linghong Zhou ${ }^{2}$, Xun Jia ${ }^{1}$, and Steve B. Jiang ${ }^{1}$ \\ Linghong Zhou: smart@smu.edu.cn; Xun Jia: xunjia@ucsd.edu; Steve B. Jiang: sbjiang@ucsd.edu \\ ${ }^{1}$ Center for Advanced Radiotherapy Technologies and Department of Radiation Medicine and \\ Applied Sciences, University of California San Diego, La Jolla, CA 92037-0843, USA \\ ${ }^{2}$ Department of Biomedical Engineering, Southern Medical University, Guangzhou, Guangdong \\ 510515, China
}

\section{Abstract}

Computed tomography (CT) to cone-beam computed tomography (CBCT) deformable image registration (DIR) is a crucial step in adaptive radiation therapy. Current intensity-based registration algorithms, such as demons, may fail in the context of CT-CBCT DIR because of inconsistent intensities between the two modalities. In this paper, we propose a variant of demons, called Deformation with Intensity Simultaneously Corrected (DISC), to deal with CT-CBCT DIR. DISC distinguishes itself from the original demons algorithm by performing an adaptive intensity correction step on the CBCT image at every iteration step of the demons registration. Specifically, the intensity correction of a voxel in CBCT is achieved by matching the first and the second moments of the voxel intensities inside a patch around the voxel with those on the CT image. It is expected that such a strategy can remove artifacts in the CBCT image, as well as ensuring the intensity consistency between the two modalities. DISC is implemented on computer graphics processing units (GPUs) in compute unified device architecture (CUDA) programming environment. The performance of DISC is evaluated on a simulated patient case and six clinical head-and-neck cancer patient data. It is found that DISC is robust against the CBCT artifacts and intensity inconsistency and significantly improves the registration accuracy when compared with the original demons.

\section{Introduction}

Online Adaptive Radiation Therapy (ART) allows real-time treatment adaptations based on the current patient anatomy and geometry. In a typical online ART process, a computed tomography (CT) image is usually acquired prior to the treatment course for treatment planning purposes. Before each treatment fraction, a cone-beam computed tomography (CBCT) image is then obtained, on which the treatment plan is redesigned to account for setup errors, deformations of tumor and other organs, as well as the change of their relative locations. Deformable image registration (DIR) technique plays an important role in this process to establish a correspondence between voxels in the $\mathrm{CT}$ and the $\mathrm{CBCT}$ for various purposes, for instance, transferring the organ contours from the planning CT images to the daily CBCT images. It is hence desirable to have an accurate and robust DIR algorithm to facilitate this step.

Among the existing DIR methods, demons (Thirion, 1998) has been proven to be a fast and robust algorithm and a number of its variants have been developed (Pennec et al., 1999; Wang et al., 2005; Rogelj and Kovacic, 2006; Yang et al., 2008). However, the demons algorithm assumes that there exists intensity consistency between two images to be registered. Therefore, although demons can successfully deal with images of the same 
modality (e.g., CT-CT registration), considerable registration error may present, when it comes to inter-modality registration problems (e.g., CT-MRI registration), where corresponding points in the two images to be registered do not necessarily pertain to the same intensity level (Guimond et al., 2001; Lu et al., 2010; Nithiananthan et al., 2011; Hou et al., 2011).

CT-CBCT DIR is considered to be an inter-modality DIR problem. Although CT and CBCT are reconstructed under the same physical principles, the intensity (Hounsfield Units (HU)) consistency between CT and CBCT images is violated due to many reasons. Firstly, almost all current commercial systems reconstruct CBCT images using the well-known FDK (Feldkamp et al., 1984) algorithm. As a fundamental limitation of this algorithm, CBCT quality degrades with increasing of cone angle (Schulze et al., 2011). Second, scatter contamination also leads to severe cupping, streak artifacts and degrade of image contrast. In a typical clinical CBCT system for radiotherapy scatter-to-primary ratio (SPR) may even exceed 100\% (Siewerdsen and Jaffray, 2001), although many methods have been proposed to correct scatter artifacts (Siewerdsen et al., 2006; Rinkel et al., 2007; Maltz et al., 2008; Zhu et al., 2009b; Poludniowski et al., 2009; Yan et al., 2010; Meyer et al., 2010; Sun et al., 2011), it is still an open problem. Third, the gantry mounted bowtie filter in CBCT system may wobble, as the gantry rotates, which can result in crescent artifacts (Giles et al., 2011; Zheng et al., 2011). Moreover, there are also other factors that contribute to the intensity inconsistency between CT and CBCT, e.g. different level of noise, beam hardening effects and motion (Zhu et al., 2009a; Hsieh et al., 2000; Grimmer and Kachelriess, 2011; Li et al., 2006; Lewis et al., 2011).

Despite the difficulties caused by the different image intensities, and the availability of many existing algorithms for multi-modal image DIR, it is still desirable to use demons-type algorithm in DIR due to its simplicity and hence high efficiency and robustness (Sharp et al., 2007; Gu et al., 2010). In our previous study (Gu et al., 2010), we had implemented 6 variants of demons algorithm and performed systematic and comprehensive evaluations which have shown that the GPU-based demons can generate accurate registration results in seconds. A variety of inter-modality demons methods have been proposed. Some researchers incorporate more reliable statistical similarity metrics into demons, such as normalized cross-correlation (NCC) or normalized mutual information (NMI), to measure the similarity between corresponding anatomical points. For instance, Modat et al (2010) implemented a diffeomorphic demons using the analytical gradient of NMI in a conjugate gradient optimizer. Lu et al (2010) has also proposed a variational approach for multimodal image registration based on the diffeomorphic demons algorithm by replacing the standard demons similarity metric with point-wise mutual information in the energy function. However, it is still unclear what alternative metrics is robust for this CT-CBCT DIR problem and how to incorporate it into demons style algorithms. On the other hand, some researchers focused on estimating the intensity relation between $\mathrm{CT}$ and CBCT images and combining intensity correction with geometrical transformation. Within this category, Guimond et al (2001) investigated the functional transformation that maps the intensities of one image to those of another, and implemented the intensity correction prior to each iteration. However, the intensity mapping of a polynomial form estimated globally based on the entire image may not be accurate enough and hence may result in errors in subsequent registration process. Recently, Hou et al (2011) attempted to correct image intensities by aligning the cumulative histograms of the two images. Nithiananthan et al (2011) also tried to correct intensities at each demons iteration by estimating linear transformations for intensity mapping for a few segmented tissue types. Nevertheless, all these works essentially assume that there exists a global mapping between the CT and the CBCT intensities, which, however, may not hold when $\mathrm{CBCT}$ is contaminated by artifacts, whose pattern is usually local. 
In this work, we propose and evaluate a modified demons algorithm embedded with a simultaneous intensity correction step, called Deformation with Intensity Simultaneously Corrected (DISC). Rather than estimating a global mathematical transformation model between CT and CBCT images, our method corrects CBCT intensity of each voxel at every iteration step of demons by matching the first and the second moments of the voxel intensities inside a patch around this voxel with those in the CT image. Quantitative evaluations of our method are performed by using both a simulation data set and six clinical head-and-neck cancer patient data sets. It is found that DISC can handle CBCT artifacts and the intensity inconsistency issue and therefore improves the registration accuracy when compared with the original demons.

\section{Methods and materials}

\subsection{The original demons algorithm}

Suppose we would like to register two images $\boldsymbol{I}_{m}(x)$ and $\boldsymbol{I}_{S}(x)$, where a vector field $v(x)$ relates these two images by $\left.\boldsymbol{I}_{m}(X+V(x))=\boldsymbol{I}_{S}(x)\right)$. Because $\boldsymbol{I}_{m}$ is gradually deformed to match $\boldsymbol{I}_{S}$, it is termed as the moving image, while $\boldsymbol{I}_{S}$ is called the static image. In the demons algorithm, the vector field $v(x)$ is solved in an iterative fashion and at each iteration the increment of the vector field $\mathrm{d} \mathbf{r}(x)$ is determined based on the image intensity at the voxel $x$. There are six different variants of the demons algorithm that have been studied by Gu et al (2010). The difference between them lies in the expression for computing $\mathrm{d} \mathbf{r}(x)$. Take the double force demons (Wang et al., 2005; Rogelj and Kovacic, 2006) as an example, the demons algorithm iteratively performs the following steps. First, calculate the increment of the moving vector (or the displacement vector) at all voxel points. Specifically, $\mathrm{d} \mathbf{r}=(\mathrm{d} x, \mathrm{~d} y, \mathrm{~d} z$ at a voxel in the double force demons is:

$$
\mathrm{d} \mathbf{r}^{(k+1)}=\frac{\left(\boldsymbol{I}_{m}^{(k)}-\boldsymbol{I}_{s}\right) \nabla \boldsymbol{I}_{s}}{\left(\boldsymbol{I}_{m}^{(k)}-\boldsymbol{I}_{s}\right)^{2}+\left|\nabla \boldsymbol{I}_{s}\right|^{2}}+\frac{\left(\boldsymbol{I}_{m}^{(k)}-\boldsymbol{I}_{s}\right) \nabla \boldsymbol{I}_{m}^{(k)}}{\left(\boldsymbol{I}_{m}^{(k)}-\boldsymbol{I}_{s}\right)^{2}+\left|\nabla \boldsymbol{I}_{m}^{(k)}\right|^{2}},
$$

where the superscript indexes the iteration step, $\boldsymbol{I}_{m}^{(k)}$ is the intensity of the moving image at the $k$ th iteration, $\boldsymbol{I}_{S}$ is the original static image. Second, smooth the resulting incremental vector field dr by convolving it with a Gaussian kernel. Third, add the incremental deformation field to the global deformation field $v(x)$ and update the moving image. This process is iteratively performed until convergence.

\subsection{The DISC algorithm}

In the context of CT-CBCT registration, the CT image is used as the moving one while the CBCT image is the static one. Because of the relative better image quality of CT, deforming $\mathrm{CT}$ yields better-controlled numerical error than deforming the CBCT. Due to the intensity inconsistency between CT and CBCT, the original demons algorithm usually fails in CTCBCT DIR. The DISC algorithm presented in this paper solves this problem by integrating a novel local intensity correction step into the demons framework. The rationale behind DISC is that, although it is generally difficult to establish a global intensity mapping between CT and $\mathrm{CBCT}$, there exists such a mapping locally at each voxel to convert the intensity from CBCT to CT. As such, we insert a CBCT intensity correction step before the first step of the original demons algorithm. This step estimates an intensity transformation at a voxel $x$ based on voxels in small cubic volumes centering at $x$ in both CT and CBCT (termed patches). Then the intensity of the CBCT image is adjusted at each voxel using the estimated voxeldependent transformations. By performing the estimation locally on small patches around each voxel, one can effectively remove image artifacts. 
Suppose at an intermediate step $k$ of the registration process, the moving image is deformed into $\boldsymbol{I}_{m}^{(k)}$. We would like to estimate two parameters $a$ and $b$ at each voxel $x$, such that the CBCT intensity $\boldsymbol{I}_{s}(x)$ is corrected into $a(x) \boldsymbol{I}_{S}(x)+b(x)$. This is achieved by comparing the patches centering at $x$ on $\boldsymbol{I}_{m}^{(k)}$ and $\boldsymbol{I}_{s}$. Let us consider two patches of size $n$ : $\mathbf{M}\left\{m_{1}, m_{2}, \cdots\right.$, $\left.m_{n}\right\}$ and $\mathbf{S}\left\{s_{1}, s_{2}, \cdots, s_{n}\right\}$, both centered at voxel $x$ in $\boldsymbol{I}_{m}^{(k)}$ and $\boldsymbol{I}_{s}$, respectively. It is our objective to find a linear intensity mapping:

$$
\mathbf{S}^{\prime}=a(x) \mathbf{S}+b(x), \quad(2)
$$

such that the intensity distributions of $\mathbf{S}^{\prime}$ are in agreement with $\mathbf{M}$. This can be achieved by matching the moments of intensity distributions of the two groups of voxels. The $p$-th order raw moment (also known as sample moment) of patch $\mathbf{X}\left\{x_{1}, x_{2}, \cdots, x_{n}\right\}$ is defined as:

$$
\mathrm{E}\left(\mathbf{X}^{p}\right)=\frac{1}{n} \sum_{i=1}^{n} x_{i}^{p} .
$$

In this work, only the first two moments are used:

$$
\mathbf{S}^{\prime}=a(x) \mathbf{S}+b(x)
$$

s.t.

$$
\begin{gathered}
\mathrm{E}(\mathbf{M})=\mathrm{E}\left(\mathbf{S}^{\prime}\right), \\
\mathrm{E}\left(\mathbf{M}^{2}\right)=\mathrm{E}\left(\mathbf{S}^{\prime 2}\right),
\end{gathered}
$$

where $a(x)$ and $b(x)$ for the voxel $x$ can be obtained by solving the above equations (see Appendix) as:

$$
\begin{aligned}
& a(x)=\mathrm{STD}(\mathbf{M}) / \mathrm{STD}(\mathbf{S}), \\
& b(x)=\mathrm{E}(\mathbf{M})-a(x) \mathrm{E}(\mathbf{S}),
\end{aligned}
$$

where $\operatorname{STD}(\cdot)$ is the standard deviation operator. Let $\mathbf{A}$ and $\mathbf{B}$ denote two vectors of length $N$ (number of voxels in $\boldsymbol{I}_{m}$ or $\boldsymbol{I}_{s}$ ) with entries $a$ and $b$, respectively. Strictly speaking, the estimation of $a(x)$ and $b(x)$ in Equations (5) and (6) are valid only when the two voxels $\boldsymbol{I}_{m}^{(k)}(x)$ and $\boldsymbol{I}_{S}(x)$ are at the same anatomical location. Yet, due to the apparent violation caused by the image deformation, this estimation is not always reliable. In practice, we only estimate those $a$ and $b$ when $\boldsymbol{I}_{m}^{(k)}(x)$ and $\boldsymbol{I}_{S}(x)$ belong to the same tissue class and limit $a$ and $b$ in a certain range to avoid false correction. Specifically, we first define a mask:

$$
\xi(x)=\left\{\begin{array}{c}
1, \text { where }\left|\boldsymbol{I}_{m}^{(k)}(x)-\boldsymbol{I}_{s}(x)\right|>\mathrm{HU}_{0} \\
1, \text { where } a(x)<a_{\min } \text { or } a(x)>a_{\text {max }} \\
0, \text { elsewhere }
\end{array}\right.
$$

where $\mathrm{HU}_{0}$ is the threshold in Hounsfield Unit to identify if two voxels in CT and CBCT images belong to the same tissue class. $a_{\min }$ and $a_{\max }$ are the lower and upper bounds of $a$. The way of choosing the parameters $\mathrm{HU}_{0}, a_{\min }$ and $a_{\max }$ will be discussed in Section 2.3. For voxels with $\xi=1$, the values of $a$ and $b$ cannot be reliably calculated using Equations (5) and (6); instead, they are estimated by interpolating/extrapolating from the $a$ and $b$ values for 
voxels with $\xi=0$. Initially, we set $a(x)=0$ and $b(x)=0$ for $\xi(x)=1$. We then estimate $a(x)$ and $b(x)$ values for voxels with $\xi=1$ by computing a weighted average of all $a$ and $b$ values of voxels inside a small cubic region $T$ centering at $x$ :

$$
\begin{gathered}
a(x)=\sum_{i \in T} \omega_{i} a_{i} / \sum_{i \in T} \omega_{i}, \\
b(x)=\sum_{i \in T} \omega_{i} b_{i} / \sum_{i \in T} \omega_{i}, \quad(9) \\
\omega_{i}=\exp \left[-\left(\boldsymbol{I}_{s}(i)-\boldsymbol{I}_{s}(x)\right)^{2} / h^{2}\right], \quad(10)
\end{gathered}
$$

where $\omega_{i}$ is the weighting factor determined by the intensity values of $\boldsymbol{I}_{s}(i)$. The underlying assumption is that voxels of similar intensities should have similar $a$ and $b$ values. $h$ is a parameter that adjusts to what extent we would like to enforce the similarity. This interpolation/extrapolation step may need to be performed for multiple times since for some voxels, the values of $\xi$ may be 1 for all voxels in their neighbor $T$. We need to point out that this interpolation/extrapolation procedure does not change the values of $a$ and $b$ for for voxels with $\xi=0$.

As soon as $a$ and $b$ are available at all voxels, an intensity correction is performed to update the image intensity of the original static image $\boldsymbol{I}_{S}(x)$ to yield an intensity corrected static image $\boldsymbol{I}_{\mathrm{S}}^{\prime}(X)=a(x) \boldsymbol{I}_{S}(X)+b(x)$, which is thus ready for the displacement calculation using the original demons algorithm. We would like to point out that any variants of the original demons can be implemented in DISC in a similar way.

\subsection{Implementation of DISC}

Before starting the DIR procedure, a global intensity transformation is first performed to shift the CBCT intensity by a constant (denoted as $\triangle \mathrm{HU}$ ) to match the mean intensities of $\mathrm{CT}$ and $\mathrm{CBCT}$. This is to correct the average HU difference between the two images to a certain extent.

Then, a multi-scale strategy is adopted so as to reduce the magnitude of the displacement with respect to voxel size. The iteration starts with the lowest resolution images, and the moving vectors obtained at a coarser level are up-sampled to serve as initial solution at a finer level. In this study, we considered two different resolution levels. Further downsampling was found not to improve registration accuracy nor efficiency.

Before the moving vector field calculation at each iteration, $\mathbf{A}$ and $\mathbf{B}$ are estimated using Equations (4) (10). Some parameters need to be first determined. We notice that $\mathrm{HU}_{0}$ is a parameter affected by the degree of intensity inconsistency and complexity of artifacts, and therefore is proportional to $\Delta \mathrm{HU}$. In this study, $\Delta \mathrm{HU}$ is about $100 \mathrm{HU}$ for simulation data, and about $100 \sim 300 \mathrm{HU}$ for clinical data. We empirically choose $\mathrm{HU}_{0}=2 \Delta \mathrm{HU}$. For $a_{\min }$ and $a_{\max }$, we can learn from Equation (5) that $a$ is actually the standard deviation ratio of the two patches. Thus, $\mathbf{A}$ is calculated before starting DIR and the median value $a_{m}$ of $\mathbf{A}$ is used to determine the range of $a$ in the subsequent steps. We let $a_{\min }=0.5 a_{m}$ and $a_{\max }=1.5 a_{m}$. As for the size of $T$ in the interpolation/extrapolation procedure to get the masked $a(x)$ and $b(x)$, we choose a relative small size $7 \times 7 \times 3$ to balance the efficiency and accuracy.

One of the most commonly used stopping criterion to judge whether the moving image has been correctly deformed to the static image is the cross correlation coefficient (Wang et al., 2005; Sharp et al., 2007; Yang et al., 2008; Samant et al., 2008). However, such a similarity 
metric does not work well in this CT-CBCT DIR context. We therefore use a convergence criterion based on the difference between successive deformation fields. We define a relative norm $X^{(k)}=\Sigma\left|\mathrm{d} \mathbf{r}^{(k+1)}\right| / \Sigma\left|\mathbf{r}^{(k)}\right|$, and use $\mathcal{K}^{(k-10)}-\mathcal{I}^{(k)} \leq \varepsilon$, where $\varepsilon=1.0 \times 10^{-4}$ as our stopping criterion. This measure is found to have a closer correspondence with spatial accuracy than correlation coefficient as DIR is stopped when there is no 'force' to push voxels any more (Gu et al., 2010).

In this work, we use the compute unified device architecture (CUDA) architecture with an NVIDIA GPU card as the implementation platform. In order to efficiently parallelize DISC in the CUDA environment, the data parallel portions of the algorithm are identified and grouped into the following kernels: 1) an intensity correction kernel to compute and interpolate $\mathbf{A}$ and $\mathbf{B} ; 2$ ) a Gaussian filter kernel to smooth images and moving vectors; 3) a gradient kernel to calculate the gradient of images; 4) a moving vector kernel to calculate and update moving vectors; 5) an interpolation kernel to deform images with moving vectors; and 6) a comparison kernel to stop the program based on the stopping criteria.

Considering all the components mentioned above, we summarize the DISC algorithm in algorithm A1:

\subsection{Evaluation}

2.4.1 Synthetic data: MC simulation-To validate our algorithm, we have generated a test dataset based on two CT images of a head-and-neck cancer patient. The first CT image is called planning CT acquired before the treatment while the second CT image was acquired half way in the treatment course for re-planning purpose and is called treatment CT here. Then, a CBCT image with realistic image artifacts is synthesized using the treatment CT image. DISC is applied to perform DIR between the planning CT image and the synthesized CBCT image and to correct the CBCT intensity. This approach offers us the ground truth for the evaluation of DISC: the treatment CT can be regarded as the scatter-free CBCT image and the deformation vector field between the planning and treatment CT images obtained using the original demons algorithm is the ground truth deformation vector field.

To synthesize a realistic CBCT image using the treatment CT image, we first convert the CT image into a digital phantom by assigning each voxel with a density value and a material type. CBCT projection images at 360 equally spaced directions covering an entire $2 \pi$ angular range are then calculated using an in-house developed software tool (called gDRR) (Jia et al., 2012) under a realistic projection geometry for a Varian Onboard-Imaging system (OBI) (Varian Medical Systems, Inc., Palo Alto, CA). In this package, the primary component in a projection image is calculated by a ray-tracing algorithm, while the scatter component is obtained by Monte Carlo simulations followed by an image smoothing process to suppress noise. Both the primary and the scatter calculations consider a variety of effects occurred in a realistic CBCT scan including the energy spectrum, the source fluence map, and the detector response, etc.. Once the projections are generated, an FDK reconstruction algorithm is invoked, yielding the CBCT image with exactly the same anatomy structures as in the treatment $\mathrm{CT}$ image but with all major CBCT artifacts such as scatter.

2.4.2 Clinical data-The performance of DISC is further assessed using clinical CT and CBCT data of six head-and-neck cancer patients. Each patient has a planning CT image and a CBCT image. The CBCT images were acquired 1-7 weeks after the first fraction of treatment on a Varian OBI system integrated in a Trilogy ${ }^{\mathrm{TM}}$ linear accelerator (Varian Medical Systems, Inc., Palo Alto, CA) using full-fan mode with a full-fan bow-tie filter on site. 
For the planning CT images, the image resolution in the transverse plane is $512 \times 512$ and the slice thickness is either 1.25 or $2.5 \mathrm{~mm}$. The pixel size in the transverse plane varies from 0.74 to $1.07 \mathrm{~mm}$. For all the CBCT images, the image size in the transverse plane is $512 \times 512$ and the slice thickness is $2.5 \mathrm{~mm}$. The pixel size in the transverse plane is 0.47 $\mathrm{mm}$.

The number of transversal slices ranges from 140 to 220 for a CT image and is approximately 70 for a CBCT image. Therefore, the field of view of the planning CT is generally larger than CBCT. The planning CT image is then cropped and re-sampled to match the dimension and resolution of the CBCT image after rigid registration. Both CT and $\mathrm{CBCT}$ images are down-sampled to half of their original size in the transverse plane. Hence, the image resolution for both CT and CBCT images after rigid registration is $256 \times 256 \times 68$ (Cases $1,2,5,6$ ) or $256 \times 256 \times 52$ (Cases 3,4 ), and the voxel size is $0.94 \times 0.94 \times 2.5 \mathrm{~mm}^{3}$.

2.4.3 Quantification of registration performance-Three similarity metrics are used in this work to quantify the DIR results. They are chosen based on two considerations. First, the metric should be observer-independent. Second, the metric should be insensitive to intensity inconsistency.

The first metric is normalized mutual information (NMI), ranging from 0 to 1 with 1 representing the highest image similarity. The second metric is called feature similarity index (FSIM) (Zhang et al., 2011; Yan et al., 2012), which tries to model the mechanism of the human visual system by capturing the main image features such as the phase congruency of the local structure and the image gradient magnitude. Detailed definition and description of FSIM are given in (Zhang et al., 2011). In this work, FSIM is calculated at each pair of corresponding transverse slices between two 3D data sets, and the average value and standard deviation are calculated. The FSIM score varies between 0 and 1 with 1 representing the most image similarity.

The third metric is the root mean squared error (RMSE) between two edge images:

$$
\operatorname{RMSE}_{\text {edge }}=\sqrt{\sum_{i}^{N}\left(\boldsymbol{I}_{1}^{\text {edge }}(i)-\boldsymbol{I}_{2}^{\text {edge }}(i)\right)^{2} / N},
$$

where $\boldsymbol{I}_{1}^{\text {edge }}(i)$ and $\boldsymbol{I}_{2}^{\text {edge }}(i)$ are the binary Canny edge images of image $\boldsymbol{I}_{1}$ and $\boldsymbol{I}_{2}$, respectively (Canny, 1986). When two images are perfectly aligned, RMSE $_{\text {edge }}$ should be zero.

\section{Results}

For clarity, following symbols are used to represent different images used in the algorithm evaluation: $\mathrm{CT}_{\text {original }}$ and $\mathrm{CBCT}_{\text {original }}$ are the $\mathrm{CT}$ and $\mathrm{CBCT}$ images before registration, respectively. $\mathrm{CT}_{\text {deformed }}^{\text {demons }}$ and $\mathrm{CT}_{\text {deformed }}^{\mathrm{DISC}}$ are the deformed $\mathrm{CT}$ images using the original demons algorithm and the DISC algorithm, respectively. $\mathrm{CBCT}_{\text {corrected }}$ is the intensity corrected CBCT image using DISC. In the simulation study, $\mathrm{CT}_{\text {original }}$ refers to the planning $\mathrm{CT}$ before registration; $\mathrm{CBCT}_{\text {original }}$ is the synthesized $\mathrm{CBCT}$ using the treatment $\mathrm{CT}$ before registration. $\mathrm{CBCT}_{\text {no artifacts }}$ is the treatment $\mathrm{CT}$ and regarded as the primary part of

$\mathrm{CBCT}_{\text {original }}$ before registration; $\mathrm{CT}_{\text {deformed }}^{\text {demon }}\left(\rightarrow \mathrm{CBCT}_{\text {no artifacts }}\right)$ and $\mathrm{CT}_{\text {deformed }}^{\text {demons }}\left(\rightarrow \mathrm{CBCT}_{\text {original }}\right)$ are the deformed $\mathrm{CT}$ images to match $\mathrm{CBCT}_{\text {no artifacts }}$ and $\mathrm{CBCT}_{\text {original }}$ using the original demons algorithm, respectively. $\mathrm{CT}_{\text {deformed }}^{\mathrm{DISC}}\left(\rightarrow \mathrm{CBCT}_{\text {original }}\right)$ is the deformed CT image to match $\mathrm{CBCT}_{\text {original }}$ using DISC. 


\subsection{Synthetic data}

Figure 1 shows the results for the simulation case. Figure 1(a) is the planning CT image before registration; Figure 1(b) is the synthesized CBCT image before registration, in which intensity variations due to scatter artifacts can be clearly observed inside the yellow dashed circle. Figure 1(c) is the scatter-free primary component of $\mathrm{CBCT}_{\text {original }}$ before registration. The comparison of vertical intensity profiles through the image center between $\mathrm{CBCT}_{\text {original }}$ and $\mathrm{CBCT}_{\text {no artifacts }}$ is shown in Figure $1(\mathrm{~g})$.

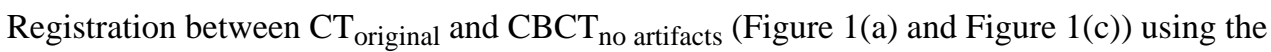
original demons algorithm can be regarded as the ground truth which yields

$\mathrm{CT}_{\text {deformed }}^{\text {demons }}\left(\rightarrow \mathrm{CBCT}_{\text {no artifacts }}\right.$ (Figure 1(d)). When the original demons algorithm is applied to deform the original CT to the original CBCT, we can see that in

$\mathrm{CT}_{\text {deformed }}^{\text {demons }}\left(\rightarrow \mathrm{CBCT}_{\text {original }}\right.$ ) (Figure 1(e)) soft tissue and bone inside the yellow dashed circle are significantly distorted after registration due to the intensity inconsistency mainly caused by scatter artifacts. We can also see the significant intensity difference between $\mathrm{CT}_{\text {deformed }}^{\text {demons }}\left(\rightarrow \mathrm{CBCT}_{\text {no artifacts }}\right)$ and $\mathrm{CT}_{\text {deformed }}^{\text {demons }}\left(\rightarrow \mathrm{CBCT}_{\text {original }}\right)$ in the intensity profile comparison in Figure 1(h). In contrast, DISC can yield correct result, as shown in Figures 1(f) and 1(h).

Checkerboard comparisons are shown in Figure 2. Misalignment is evident before registration (Figures 2(a)-1 3). The original demons algorithm unrealistically distorts the tissues after registration, especially in those central regions with severe scatter artifacts (Figures 2(b)-1 3). Results of DISC are shown in Figures 2(c)-1 3 and Figures 2(d)-1 3. As we can see, DISC is robust against the artifacts and able to match the two images well without distortion (Figure 2(c)-1 3). Checkerboard boundary between $\mathrm{CBCT}_{\text {corrected }}$ and $\mathrm{CT}_{\text {deformed }}^{\mathrm{DISC}}\left(\rightarrow \mathrm{CBCT}_{\text {original }}\right)$ can hardly be seen since $\mathrm{CBCT}_{\text {corrected }}$ has similar intensity distribution as the deformed CT (Figure 2(d)-1 3).

The improvement of DISC over original demons can be further examined by inspecting the difference of the moving vector fields (Figure 3). Because of the absence of artifacts in $\mathrm{CBCT}_{\text {no artifacts }}$, it is expected that the original demons algorithm is functional when it is used for DIR between $\mathrm{CT}_{\text {original }}$ and $\mathrm{CBCT}_{\text {no artifacts, }}$, and the resulting deformation vector field $\mathbf{r}_{\mathbf{0}}$ can be regarded as the ground truth vector field. We further denote the vector field obtained by the original demons algorithm and DISC between $\mathrm{CT}_{\text {original }}$ and $\mathrm{CBCT}_{\text {original }}$ by $\mathbf{r}_{1}$ and $\mathbf{r}_{2}$. The errors in terms of vector field in these two cases are hence characterized by the difference between $\left|\mathbf{r}_{\mathbf{1}}-\mathbf{r}_{\mathbf{0}}\right|$ and $\left|\mathbf{r}_{\mathbf{2}}-\mathbf{r}_{\mathbf{0}}\right|$, which are shown in Figure 3. We can see that the original demons produces incorrect deformation vector field when artifacts exist (Figure 3(a)), while the DISC algorithm can yield almost the same deformation vector field as the ground truth (Figure 3(b)).

On the other hand, the efficacy of CBCT intensity correction is demonstrated in Figure 4, where difference images between $\mathrm{CBCT}_{\text {no artifacts }}$ and $\mathrm{CBCT}_{\text {original }}$, and between $\mathrm{CBCT}_{\text {no artifacts }}$ and $\mathrm{CBCT}_{\text {corrected }}$ are shown. $\mathrm{CBCT}_{\text {no artifacts }}$ is considered as the CBCT image without any artifacts and thus with correct intensity. Before DIR, the scatter artifact is evident in $\mathrm{CBCT}_{\text {original }}$ and hence a large deviation is observed from $\mathrm{CBCT}_{\text {no artifacts }}$ (Figure 4(a)). As DISC proceeds, the CBCT intensity is corrected gradually and the $\mathrm{CBCT}_{\text {corrected }}$ is resulted, which has relatively small intensity inconsistency when compared with $\mathrm{CBCT}_{\text {no artifacts }}$ (Figure 4(b)). 


\subsection{Clinical cases}

The performance of the DISC algorithm is further assessed on six head-and-neck cancer patient cases. An example result (Case 2) is shown in Figures 5 and 6. Misalignment is evident before registration (Figures 6 (a)-1 3). The results from the original demons algorithm are shown in Figure 5(c) and Figures 6(b)-1 3. As we can see, the anatomical structures in the deformed CT image are significantly distorted due to intensity inconsistency. This effect is more severe in regions that are dominated by artifacts (as indicated by arrows in Figure 5(c) and Figures 6(b)-1 3). The DISC algorithm (Figure 5(d) and Figures 6(c)-1 3), on the other hand, yields undistorted CT image that matches well with the original CBCT, which can also be seen from the comparison between $\mathrm{CT}_{\text {deformed }}^{\mathrm{DISC}}$ and $\mathrm{CBCT}_{\text {corrected }}$ as shown in Figures 6(d)-1 3. This can be also verified by examining the histograms of $\mathrm{CT}_{\text {original }}, \mathrm{CBCT}_{\text {original }}, \mathrm{CT}_{\text {deformed }}^{\mathrm{DISC}}$ and $\mathrm{CBCT}_{\text {corrected }}$ (Figure 7). We can see the remarkable histogram difference between $\mathrm{CT}_{\text {original }}$ and $\mathrm{CBCT}_{\text {original }}$ before DISC, and almost the same histogram distributions between $\mathrm{CT}_{\text {deformed }}^{\mathrm{DISC}}$ and $\mathrm{CBCT}_{\text {corrected. This }}$ indicates that the patch-based DISC algorithm can achieve a global match of intensity distribution, although the operations are purely local at each voxel.

In terms of DIR accuracy, the DISC algorithm is quantitatively evaluated using NMI, FSIM and Canny edge RMSE between $\mathrm{CT}_{\text {original }}$ and $\mathrm{CBCT}_{\text {original }}, \mathrm{CT}_{\text {deformed }}^{\text {demos }}$ and $\mathrm{CBCT}_{\text {original }}$,

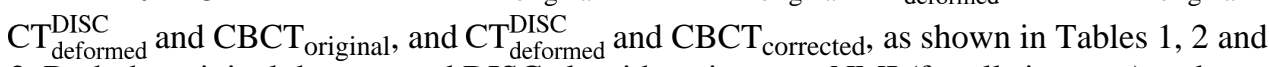
3. Both the original demons and DISC algorithms increase NMI (for all six cases) and reduce Canny edge RMSE (except for case 3 and 6 where the original demons algorithm generates even larger Canny edge RMSE) between CT and CBCT images. The FSIM for all six cases are increased after DISC. Interestingly, except for Case 2, the FSIM is decreased after DIR using the original demons algorithm. This is because the original demons algorithm distorts tissues significantly after DIR, which can be easily observed by human visual inspection but hard to be detected by NMI or Canny edge RMSE. It is much easier for FSIM to detect such changes, since it scores the similarity by mimicking how the human vision works. For all six cases, the average NMI increases from $0.62 \pm 0.02$ to $0.63 \pm 0.02$, the average FSIM increases from $0.91 \pm 0.04$ to $0.94 \pm 0.02$, and the average edge RMSE decreases from $0.24 \pm 0.03$ to $0.21 \pm 0.03$, when comparing DISC with the original demons algorithm.

\subsection{Effect of patch size}

The patch size in DISC has a considerable impact on the registration performance. Figures 8(a)-(l) show part of the $\mathrm{CT}_{\text {deformed }}^{\text {DISC }}$ with different patch sizes in an example clinical case. This part of image is contaminated by artifacts most severely. As the patch size increases, the bones become distorted and the edges become blurred. When the patch size gets even larger, soft tissue region is distorted as well (Figures 8(g)-(l)). This effect is also observed in other clinical cases. The reason is that, as the patch size increases, it is more likely to have different structures included in the same patch, resulting in errors in intensity correction. Figure 9(a)-(l) show part of the $\mathrm{CBCT}_{\text {corrected }}$ with different patch sizes of the same case. We can also observe that as the patch size increases, the image quality of $\mathrm{CBCT}_{\text {corrected }}$ decreases. Therefore, we use $3 \times 3 \times 3$ patch size in this work for all simulation data and clinical data, and the patch size is kept constant at each image resolution level.

\subsection{Variable $a$ and $b$}

Figures 10(a) and 10(b) show one transversal slice of the distribution of the parameters $a$ and $b$ at the last iteration of DISC. Figures 10(c) and 10(d) are the patch standard deviation in $\boldsymbol{I}_{m}$ and $\boldsymbol{I}_{s}$ at the last iteration of DISC. In fact, $a$ is the ratio of the pixel value in Figure 10(c) to 
that in Figure 10(d) (see Equation (5)), and it controls the slope of the intensity transformation in each voxel. The value of $b$ represents a shift of the mean intensity value in a patch in $\boldsymbol{I}_{S}$ to match that in $\boldsymbol{I}_{m}$. We can see that the region with relatively high values of $b$ indicated by an arrow in Figure 10(b), showing a large shift is applied to change the mean intensity value of the patch, corresponds to the region where the large artifacts present in the original CBCT image. Figures 10(e) and 10(f) depict the evolution of the average value of $a$ and $b$ during the DISC iteration. We can see that both $a$ and $b$ converge nicely at both image resolution levels.

\subsection{Computational efficiency}

All the experiments in this study were conducted on an NVIDIA Telsa C1060 card with a total number of 240 processors of $1.3 \mathrm{GHz}$. It is also equipped with 4 GB DDR3 memory, shared by all processors. In the CUDA implementation of intensity correction kernel in DISC, the computation corresponding to each voxel is handled by a thread, which loops over all voxels in the patch centered at the voxel. Consequently, the computational time is highly dependent on the patch size. The most time consuming step in the calculation is the iterative interpolation/extrapolation procedure to get $a$ and $b$ values for voxels with $\xi=1$. Currently, there is no efficient way for sparse data interpolation. Compared to $\sim 20$ s needed with the original demons algorithm, the DISC algorithm takes about $69 \mathrm{~s}$ for a patch size of $3 \times 3 \times 3$ and an image size of $256 \times 256 \times 68$.

\section{Discussion and Conclusions}

A fast, accurate, and robust CT-CBCT DIR algorithm is a key step in ART. Though many methods had been studied specifically for inter-modality image registration, which might be applied directly to CT-CBCT DIR, it is still tempting to use a more accurate and efficient intensity-based algorithm, such as demons, to deal with the DIR problem for CT and CBCT images. Other than modifying the DIR similarity metric, which is not straightforward in demons, we presented in this paper a patch-based intensity correction method that is performed in conjunction with demons, namely DISC, and evaluated it comprehensively with simulated data and clinical patient data. By incorporating the intensity correction at every iteration step, the DISC algorithm can robustly estimate the spatial transformation while guaranteeing the intensity consistency between CT and CBCT image.

It is technically possible to use different patch sizes at different parts of the image. One practical approach is to adjust the patch size according to the local image content. For instance, a relatively large patch size can be used in those homogeneous regions, while a small patch size can be adopted in those regions with more image details. The patch size can be thus determined adaptively by calculating the standard deviation of a specific region, which might help the convergence of the algorithm. This idea will be studied in the future work.

Note that in Case 2, the $\mathrm{CT}_{\text {original }}$ has been truncated. This is because the CBCT has a limited field of view (FOV) due to the limited size of the image detector. In this work, we first rigidly register the CT with this truncated CBCT and then crop the CT outside the FOV, which results in a cropped CT image after registration. Undoubtedly, this cropped CT image cannot be used for dose calculation afterward in ART, as it may introduce significant errors. This truncation problem is not unique to DISC but a common issue for all DIR algorithms. Yang et al (2010) proposed to assign those missing voxels outside of the FOV with $\mathrm{NaN}$ (not-a-number) value. This simple method makes it possible to avoid the cropping of the CT image. We are developing a more mathematically strict method to modify DISC for the DIR problem between CT image and truncated CBCT image. 
Most previous studies focused on CBCT images without severe artifacts, in which case simple histogram matching (Hou et al., 2011) or polynomial transformation model (Nithiananthan et al., 2011) might be sufficient to describe the intensity relationship between the $\mathrm{CT}$ and CBCT image. In our DISC algorithm, intensity correction is performed on a voxel-by-voxel basis and the correction parameters are estimated by considering the voxels nearby. It is advantageous to do so, because usually, obtaining an ideal mathematical model to describe the intensity relation is not easy, if not impossible, when considering the existence of severe artifacts in CBCT images. While estimating the intensity transformation locally, one is able to make use of the prior information from CT image, and correct the intensity in CBCT image voxel-wise. By this means, we try to equalize the intensities distribution (the sample moments) inside each small patch with its corresponding patch in the other image, which is physically sensible. Embedding this additional correction step into the original demons, the intensity consistency requirement can be guaranteed.

Another merit of the DISC method is that it can yield the intensity corrected CBCT image at the end of the DIR. This intensity corrected CBCT image can be regarded as a CBCT image produced by the primary radiation. Hence, the DISC method, potentially, can be applied to the area of CBCT scatter removal and hence other clinically relevant tasks, e.g. radiation dose calculation based on CBCT.

The intensity correction method in DISC can be easily incorporated into other intensitybased DIR algorithms, such as optical flow based algorithms (Lucas and Kanade, 1981), fast free form with calculus of variations (Lu et al., 2004), etc., where intensity consistency between two images is assumed. This method is effective in correcting the intensity of CBCT images because of the modality similarity between CT and CBCT images. For more challenging multi-modality DIR tasks, such as CT to MR DIR, our intensity correction method needs to be modified since same anatomical structures in the MR image may have totally different or even opposite image intensity with those in the CT image.

Another potential application of this intensity correction method might be in the field of image segmentation. Medical images often suffer from various imaging artifacts and the image intensity may not be homogeneous in regions where image homogeneity should exist. This issue may result in the failure of some intensity-driven segmentation methods (e.g., $\mathrm{Li}$ et al., 2011; Shahvaran et al., 2012). To solve this problem, our intensity correction method may be incorporated into a more sophisticated framework based on the combination of simultaneous registration, segmentation, and intensity correction using artifact-free prior images.

\section{Acknowledgments}

This work is supported in part by NIH (1R01CA154747-01), the University of California Lab Fees Research Program, Varian Medical Systems, Inc., and the National Natural Science Foundation of China (No. 30970866)

\section{References}

Canny J. A Computational Approach to Edge Detection. Pattern Analysis and Machine Intelligence, IEEE Transactions on PAMI-8. 1986:679-98.

Feldkamp LA, Davis LC, Kress JW. Practical cone-beam algorithm. J Opt Soc Am A. 1984; 1:612-9.

Giles W, Bowsher J, Li H, Yin F-F. Crescent artifacts in cone-beam CT. Medical Physics. 2011; 38:2116. [PubMed: 21626944]

Grimmer R, Kachelriess M. Empirical binary tomography calibration (EBTC) for the precorrection of beam hardening and scatter for flat panel CT. Medical Physics. 2011; 38:2233-40. [PubMed: 21626957] 
Gu X, Pan H, Liang Y, Castillo R, Yang D, Choi D, Castillo E, Majumdar A, Guerrero T, Jiang SB. Implementation and evaluation of various demons deformable image registration algorithms on a GPU. Physics in Medicine and Biology. 2010; 55:207-19. [PubMed: 20009197]

Guimond A, Roche A, Ayache N, Meunier J. Three-dimensional multimodal brain warping using the demons algorithm and adaptive intensity corrections. IEEE Trans Med Imaging. 2001; 20:58-69. [PubMed: 11293692]

Hou J, Guerrero M, Chen W, D’Souza WD. Deformable planning CT to cone-beam CT image registration in head-and-neck cancer. Medical Physics. 2011; 38:2088. [PubMed: 21626941]

Hsieh J, Molthen RC, Dawson CA, Johnson RH. An iterative approach to the beam hardening correction in cone beam CT. Medical Physics. 2000; 27:23-9. [PubMed: 10659734]

Jia X, Yan H, Cervino L, Folkerts M, Jiang SB. A GPU Tool for Efficient, Accurate, and Realistic Simulation of Cone Beam CT Projections. 2012 submitted to Med Phys.

Lewis JH, Li R, Jia X, Watkins WT, Lou Y, Song WY, Jiang SB. Mitigation of motion artifacts in CBCT of lung tumors based on tracked tumor motion during CBCT acquisition. Physics in Medicine and Biology. 2011; 56:5485-502. [PubMed: 21813959]

Li T, Schreibmann E, Yang Y, Xing L. Motion correction for improved target localization with onboard cone-beam computed tomography. Physics in Medicine and Biology. 2006; 51:253-67. [PubMed: 16394337]

Li Y, Hoover-Fong J, Carrino JA, Mori S. Simultaneous segmentation and inhomogeneity correction in magnetic resonance images. Conf Proc IEEE Eng Med Biol Soc. 2011; 2011:8045-8. [PubMed: 22256208]

Lu, H.; Reyes, M.; Serijovic, A.; Weber, S.; Sakurai, Y.; Yamagata, H.; Cattin, PC. Proceedings of the 2010 IEEE international conference on Biomedical imaging: from nano to Macro. Rotterdam, Netherlands: IEEE Press; 2010. Multi-modal diffeomorphic demons registration based on pointwise mutual information; p. 372-5.

Lu W, Chen M-L, Olivera GH, Ruchala KJ, Mackie TR. Fast free-form deformable registration via calculus of variations. Physics in Medicine and Biology. 2004; 49:3067-87. [PubMed: 15357182]

Lucas, BD.; Kanade, T. An iterative image registration technique with an application to stereo vision. Proceedings of the 7th international joint conference on Artificial intelligence; Vancouver, BC, Canada: Morgan Kaufmann Publishers Inc; 1981. p. 674-9.

Maltz JS, Gangadharan B, Bose S, Hristov DH, Faddegon BA, Paidi A, Bani-Hashemi AR. Algorithm for X-ray scatter, beam-hardening, and beam profile correction in diagnostic (kilovoltage) and treatment (megavoltage) cone beam CT. IEEE Trans Med Imaging. 2008; 27:1791-810. [PubMed: 19033095]

Meyer M, Kalender WA, Kyriakou Y. A fast and pragmatic approach for scatter correction in flatdetector CT using elliptic modeling and iterative optimization. Phys Med Biol. 2010; 55:99-120. [PubMed: 20009184]

Modat, M.; Vercauteren, T.; Ridgway, GR.; Hawkes, DJ.; Fox, NC.; Ourselin, S. Diffeomorphic demons using normalized mutual information, evaluation on multimodal brain MR images. Dawant, BM.; Haynor, DR., editors. San Diego, California, USA: SPIE; 2010. p. 76232K-8

Nithiananthan S, Schafer S, Uneri A, Mirota DJ, Stayman JW, Zbijewski W, Brock KK, Daly MJ, Chan H, Irish JC, Siewerdsen JH. Demons deformable registration of CT and cone-beam CT using an iterative intensity matching approach. Medical Physics. 2011; 38:1785. [PubMed: 21626913]

Pennec, X.; Cachier, P.; Ayache, N. Understanding the "Demon's Algorithm": 3D Non-Rigid registration by Gradient Descent. 2nd int. conf. on medical image computing and computerassisted intervention (MICCAI'99) LNSC; 1999. p. 597-605.

Poludniowski G, Evans PM, Hansen VN, Webb S. An efficient Monte Carlo-based algorithm for scatter correction in keV cone-beam CT. Physics in Medicine and Biology. 2009; 54:3847. [PubMed: 19491449]

Rinkel J, Gerfault L, Estève F, Dinten JM. A new method for x-ray scatter correction: first assessment on a cone-beam CT experimental setup. Physics in Medicine and Biology. 2007; 52:4633. [PubMed: 17634655]

Rogelj P, Kovacic S. Symmetric image registration. Med Image Anal. 2006; 10:484-93. [PubMed: 15896998] 
Samant SS, Xia J, Muyan-Özçelik P, Owens JD. High performance computing for deformable image registration: Towards a new paradigm in adaptive radiotherapy. Medical Physics. 2008; 35:3546. [PubMed: 18777915]

Schulze R, Heil U, Gross D, Bruellmann D, Dranischnikow E, Schwanecke U, Schoemer E. Artefacts in CBCT: a review. Dentomaxillofacial Radiology. 2011; 40:265-73. [PubMed: 21697151]

Shahvaran Z, Kazemi K, Helfroush MS, Jafarian N, Noorizadeh N. Variational level set combined with Markov random field modeling for simultaneous intensity non-uniformity correction and segmentation of MR images. Journal of Neuroscience Methods. 2012

Sharp GC, Kandasamy N, Singh H, Folkert M. GPU-based streaming architectures for fast cone-beam CT image reconstruction and demons deformable registration. Physics in Medicine and Biology. 2007; 52:5771-83. [PubMed: 17881799]

Siewerdsen JH, Daly MJ, Bakhtiar B, Moseley DJ, Richard S, Keller H, Jaffray DA. A simple, direct method for x-ray scatter estimation and correction in digital radiography and cone-beam CT. Med Phys. 2006; 33:187-97. [PubMed: 16485425]

Siewerdsen JH, Jaffray DA. Cone-beam computed tomography with a flat-panel imager: Magnitude and effects of x-ray scatter. Medical Physics. 2001; 28:220. [PubMed: 11243347]

Sun M, Nagy T, Virshup G, Partain L, Oelhafen M, Star-Lack J. Correction for patient table-induced scattered radiation in cone-beam computed tomography (CBCT). Medical Physics. 2011; 38:2058-73. [PubMed: 21626939]

Thirion JP. Image matching as a diffusion process: an analogy with Maxwell's demons. Med Image Anal. 1998; 2:243-60. [PubMed: 9873902]

Wang H, Dong L, O’Daniel J, Mohan R, Garden AS, Ang KK, Kuban DA, Bonnen M, Chang JY, Cheung R. Validation of an accelerated 'demons' algorithm for deformable image registration in radiation therapy. Physics in Medicine and Biology. 2005; 50:2887-905. [PubMed: 15930609]

Yan H, Cervino L, Jia X, Jiang SB. A comprehensive study on the relationship between the image quality and imaging dose in low-dose cone beam CT. Physics in Medicine and Biology. 2012; 57:2063-80. [PubMed: 22459913]

Yan H, Mou X, Tang S, Xu Q, Zankl M. Projection correlation based view interpolation for cone beam CT: primary fluence restoration in scatter measurement with a moving beam stop array. Physics in Medicine and Biology. 2010; 55:6353-75. [PubMed: 20938067]

Yang D, Goddu SM, Lu W, Pechenaya OL, Wu Y, Deasy JO, El Naqa I, Low DA. Technical Note: Deformable image registration on partially matched images for radiotherapy applications. Medical Physics. 2010; 37:141. [PubMed: 20175475]

Yang D, Li H, Low DA, Deasy JO, Naqa IE. A fast inverse consistent deformable image registration method based on symmetric optical flow computation. Physics in Medicine and Biology. 2008; 53:6143-65. [PubMed: 18854610]

Zhang L, Zhang L, Mou X, Zhang D. FSIM: a feature similarity index for image quality assessment. IEEE Trans Image Process. 2011; 20:2378-86. [PubMed: 21292594]

Zheng D, Ford JC, Lu J, Lazos D, Hugo GD, Pokhrel D, Zhang L, Williamson JF. Bow-tie wobble artifact: Effect of source assembly motion on cone-beam CT. Medical Physics. 2011; 38:2508-14. [PubMed: 21776785]

Zhu L, Wang J, Xing L. Noise suppression in scatter correction for cone-beam CT. Med Phys. 2009a; 36:741-52. [PubMed: 19378735]

Zhu L, Xie Y, Wang J, Xing L. Scatter correction for cone-beam CT in radiation therapy. Medical Physics. 2009b; 36:2258. [PubMed: 19610315]

\section{Appendix. Derivation of Equations (5) and (6)}

Given two groups of points $\mathbf{M}\left\{m_{1}, m_{2}, \cdots, m_{\mathrm{n}}\right\}$ and $\mathbf{S}\left\{s_{1}, s_{2}, \cdots, s_{\mathrm{n}}\right\}$, it is our objective to match the first and the second moments after a linear transformation. Specifically, we seek for constants $a(x)$ and $b(x)$ for the intensity transformation 


$$
\mathbf{S}^{\prime}=a(x) \mathbf{S}+b(x), \quad(\text { A.1 })
$$

s.t.

$$
\begin{gathered}
\mathrm{E}(\mathbf{M})=\mathrm{E}\left(\mathbf{S}^{\prime}\right), \\
\mathrm{E}\left(\mathbf{M}^{2}\right)=\mathrm{E}\left(\mathbf{S}^{\prime 2}\right)
\end{gathered}
$$

Because these two equations lead to

$$
\operatorname{STD}(\mathbf{M})=\sqrt{\mathrm{E}\left(\mathbf{M}^{2}\right)-(\mathrm{E}(\mathbf{M}))^{2}}=\sqrt{\mathrm{E}\left(\mathbf{S}^{\prime 2}\right)-\left(\mathrm{E}\left(\mathbf{S}^{\prime}\right)\right)^{2}}=\operatorname{STD}\left(\mathbf{S}^{\prime}\right), \quad \text { (A.4) }
$$

taking the standard deviations on both sides of (A.1) yields

$$
\operatorname{STD}(\mathbf{M})=\operatorname{STD}(a(x) \mathbf{S}+b(x))=a(x) \operatorname{STD}(\mathbf{S}) \quad(\text { A.5 })
$$

Hence

$$
\begin{gathered}
a(x)=\mathrm{STD}(\mathbf{M}) / \operatorname{STD}(\mathbf{S}), \quad \text { (A.6) } \\
b(x)=\mathrm{E}(\mathbf{M})-a(x) \mathrm{E}(\mathbf{S}) . \quad(\text { A.7) }
\end{gathered}
$$



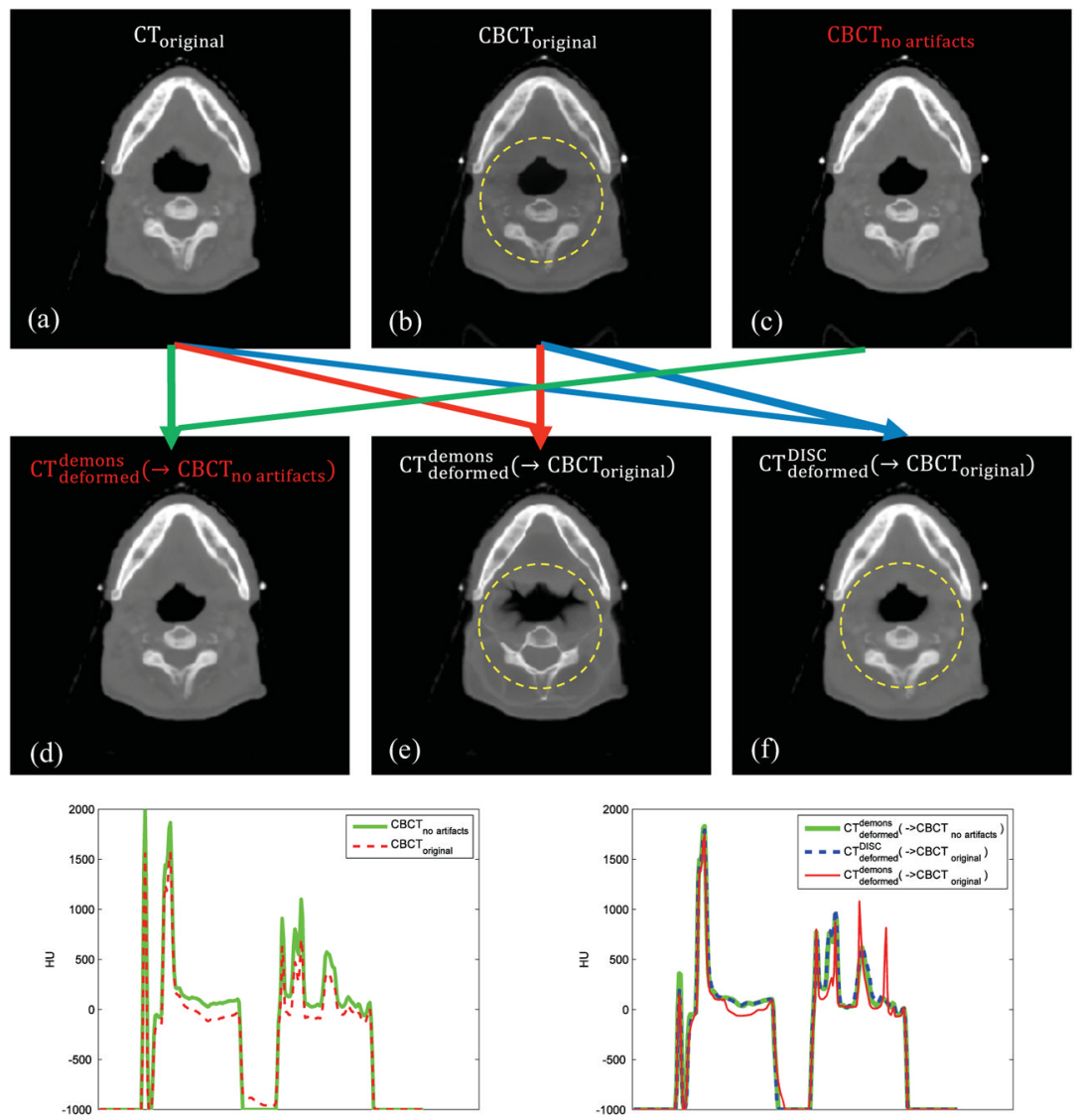

(g)

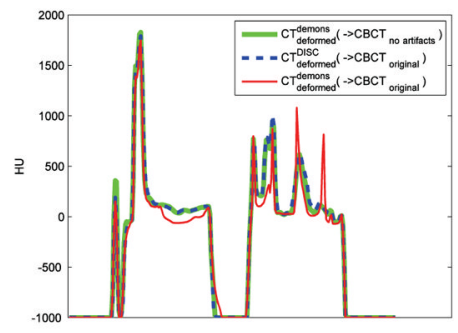

(h)

Figure 1.

Simulation and registration results. The yellow dashed circles indicate the regions which severely suffer from the scatter artifact. The green arrow indicates the DIR between $\mathrm{CT}_{\text {original }}$ and $\mathrm{CBCT}_{\text {no artifacts }}$ using the original demons algorithm; the red arrow indicates the DIR between $\mathrm{CT}_{\text {original }}$ and $\mathrm{CBCT}_{\text {original }}$ using the original demons algorithm; the blue arrow indicates the DIR between $\mathrm{CT}_{\text {original }}$ and $\mathrm{CBCT}_{\text {original }}$ using DISC. $(\mathrm{g})$ and $(\mathrm{h})$ are the corresponding vertical intensity profiles through the image center. 

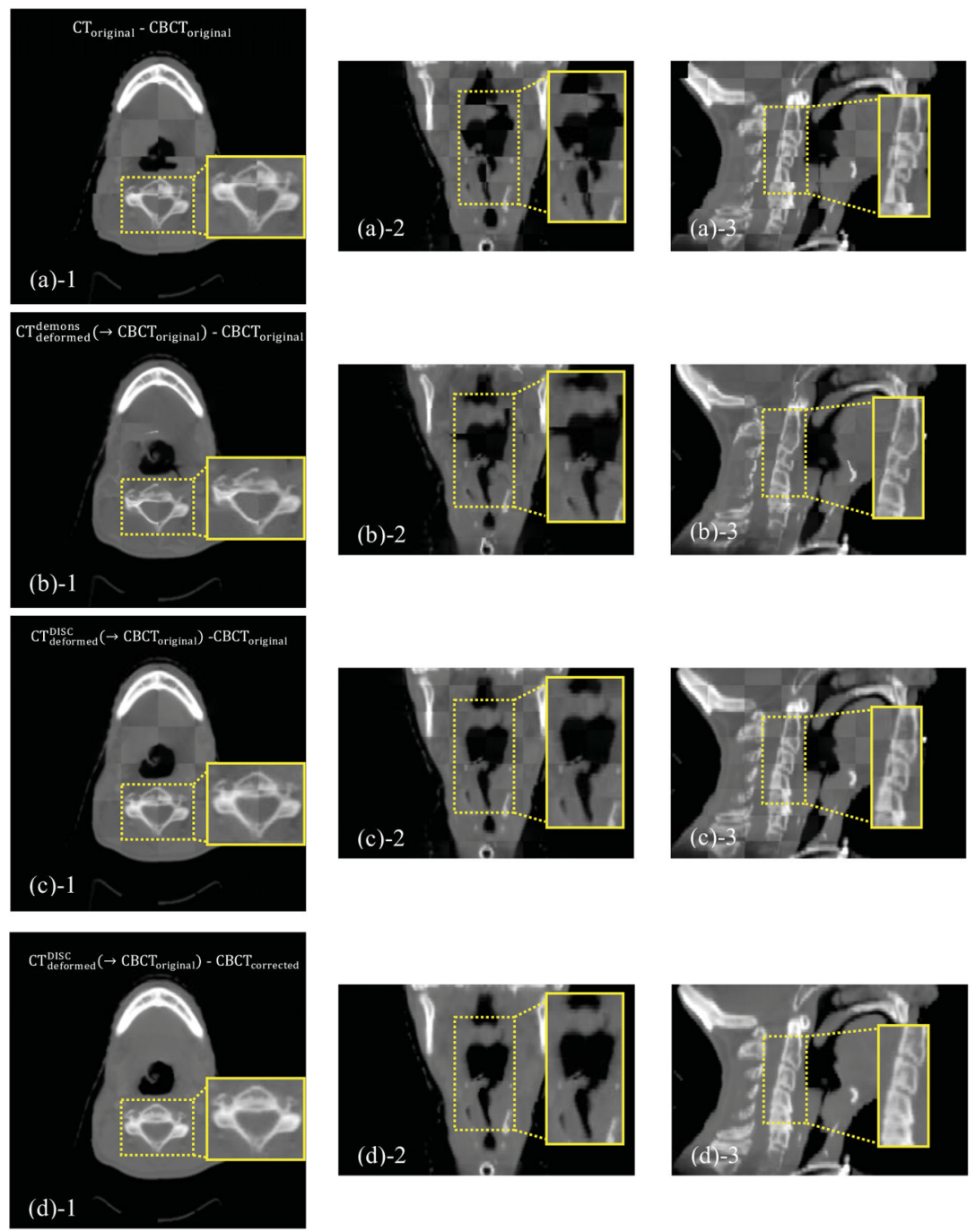

Figure 2.

Checkerboard comparisons of the DIR results based on the simulation dataset. Columns: transverse, coronal and sagittal images, respectively. (a): $\mathrm{CT}_{\text {original }}$ and $\mathrm{CBCT}_{\text {original }}$; (b): $\mathrm{CT}_{\text {deformed }}^{\text {demons }}\left(\rightarrow \mathrm{CBCT}_{\text {original }}\right)$ and $\mathrm{CBCT}_{\text {original }} ;(\mathrm{c}): \mathrm{CT}_{\text {deformed }}^{\mathrm{DISC}}\left(\rightarrow \mathrm{CBCT}_{\text {original }}\right)$ and $\mathrm{CBCT}_{\text {original }} ;(\mathrm{d}): \mathrm{CT}_{\text {deformed }}^{\mathrm{DISC}}\left(\rightarrow \mathrm{CBCT}_{\text {original }}\right)$ and $\mathrm{CBCT}_{\text {corrected }}$ The insets show the zoomed-in views. 

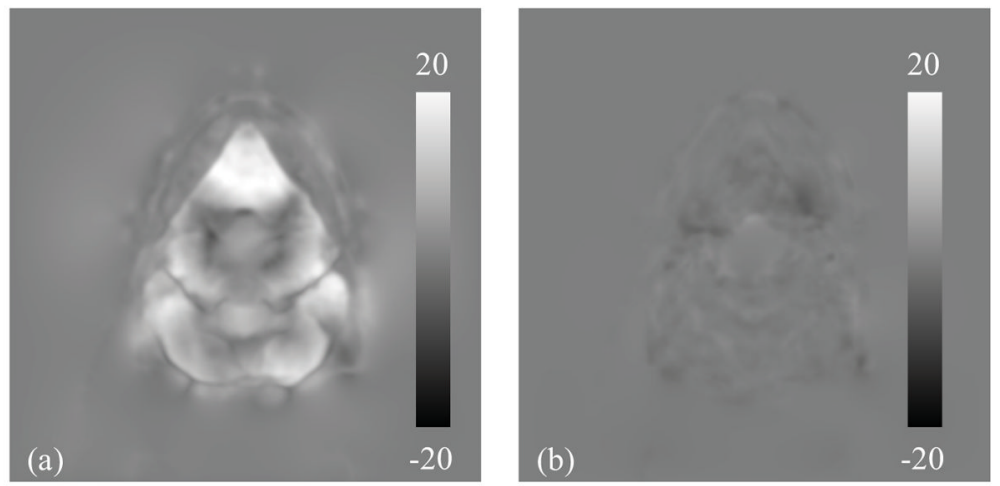

Figure 3.

Difference of the deformation vector fields (display window: -20 20 voxels): (a): between $\mathrm{CT}_{\text {deformed }}^{\text {demons }}\left(\rightarrow \mathrm{CBCT}_{\text {original }}\right)$ and $\mathrm{CT}_{\text {deformed }}^{\text {demons }}\left(\rightarrow \mathrm{CBCT}_{\text {no artifacts }}\right) ;(\mathrm{b})$ : between $\mathrm{CT}_{\text {deformed }}^{\mathrm{DISC}}\left(\rightarrow \mathrm{CBCT}_{\text {original }}\right)$ and $\mathrm{CT}_{\text {deformed }}^{\text {demons }}\left(\rightarrow \mathrm{CBCT}_{\text {no artifacts }}\right)$. 

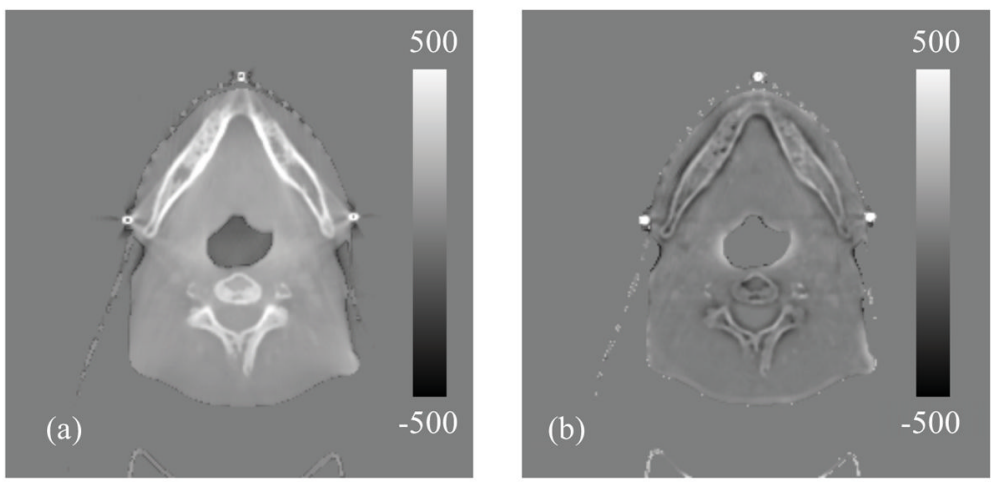

Figure 4.

Difference images (display window: $-500 \sim 500 \mathrm{HU}$ ): (a) between $\mathrm{CBCT}_{\text {no artifacts }}$ and $\mathrm{CBCT}_{\text {original }}$; (b) between $\mathrm{CBCT}_{\text {no artifacts }}$ and $\mathrm{CBCT}_{\text {corrected }}$. 

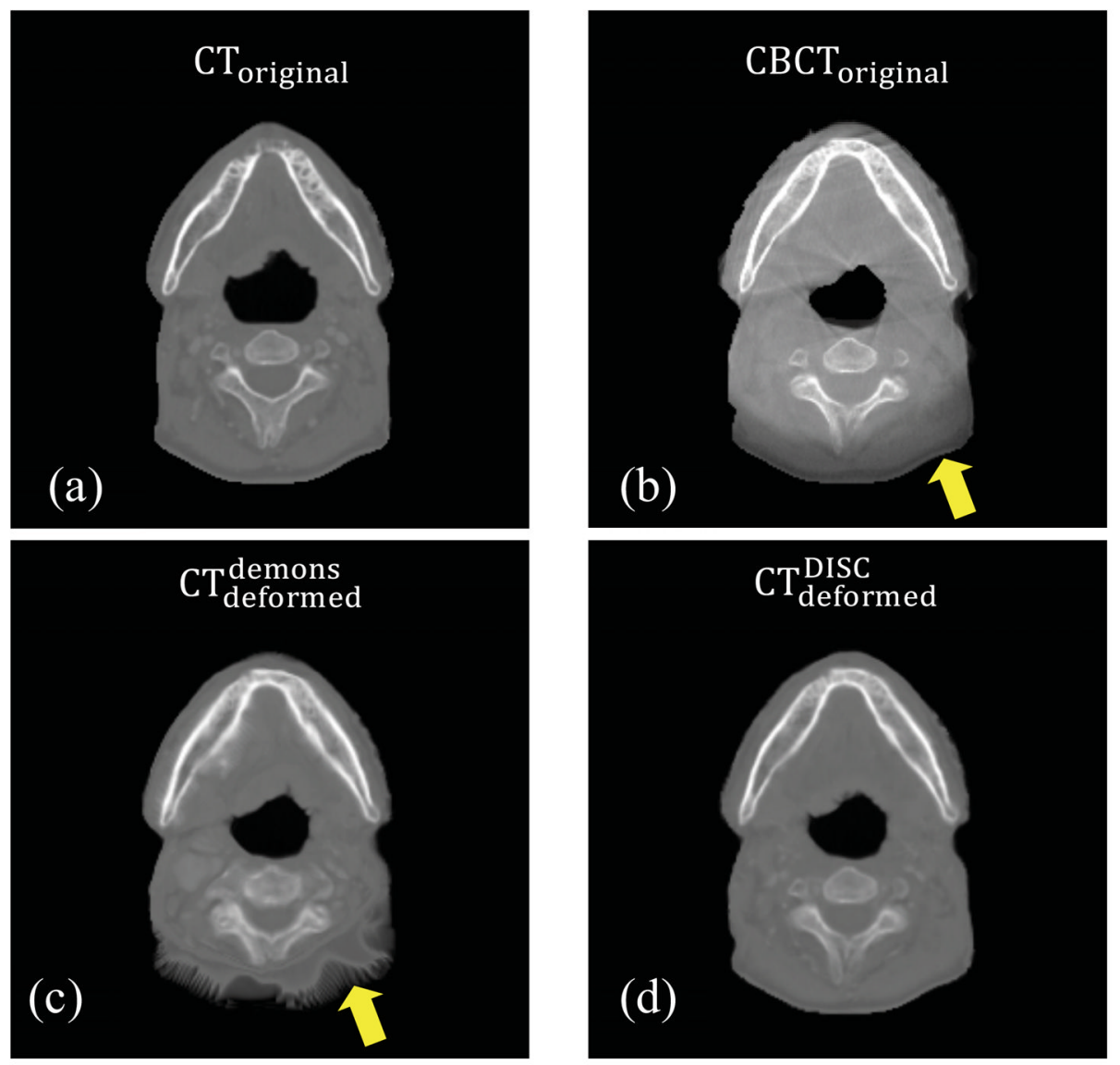

Figure 5.

(a): $\mathrm{CT}_{\text {original }}$; (b): $\mathrm{CBCT}_{\text {original }}$; (c): $\mathrm{CT}_{\text {deformed }}^{\text {demons }}$ (d): $\mathrm{CT}_{\text {deformed }}^{\mathrm{DISC}}$. The arrows indicate the region contaminated by artifacts (b) and the region with geometrical distortion (c). 

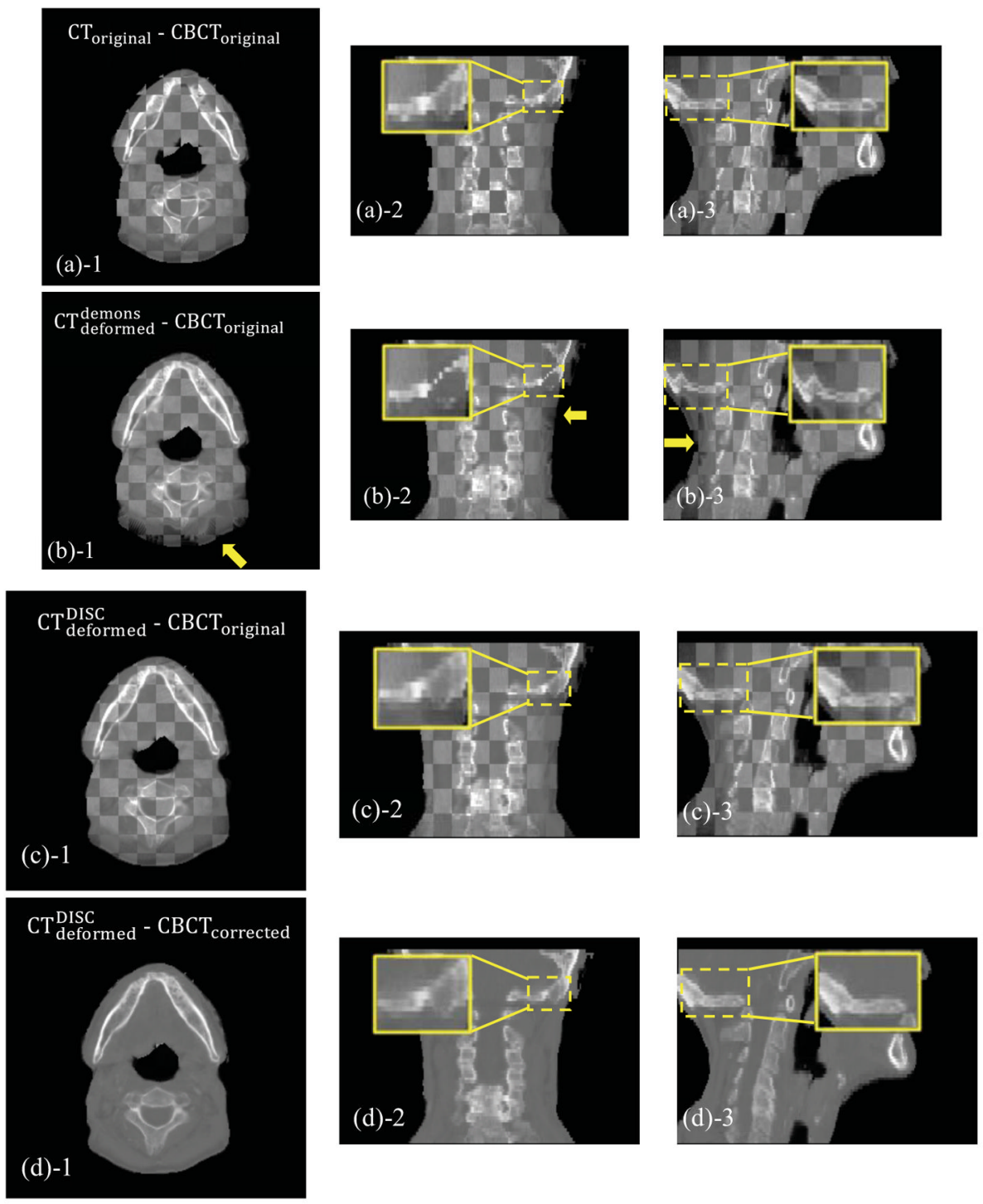

Figure 6.

Checkerboard comparisons of Case 2. Columns: transverse, coronal and sagittal images, respectively. (a): $\mathrm{CT}_{\text {original }}$ and $\mathrm{CBCT}_{\text {original }}$; (b): $\mathrm{CT}_{\text {deformed }}^{\text {demons }}$ and $\mathrm{CBCT}_{\text {original }}$; (c): $\mathrm{CT}_{\text {deformed }}^{\mathrm{DISC}}$ and $\mathrm{CBCT}_{\text {original }}$; (d): $\mathrm{CT}_{\text {deformed }}^{\mathrm{DISC}}$ and $\mathrm{CBCT}_{\text {corrected }}$ The insets show the zoomed-in views, and the arrows indicate the regions contaminated by artifact or with geometrical distortion. 


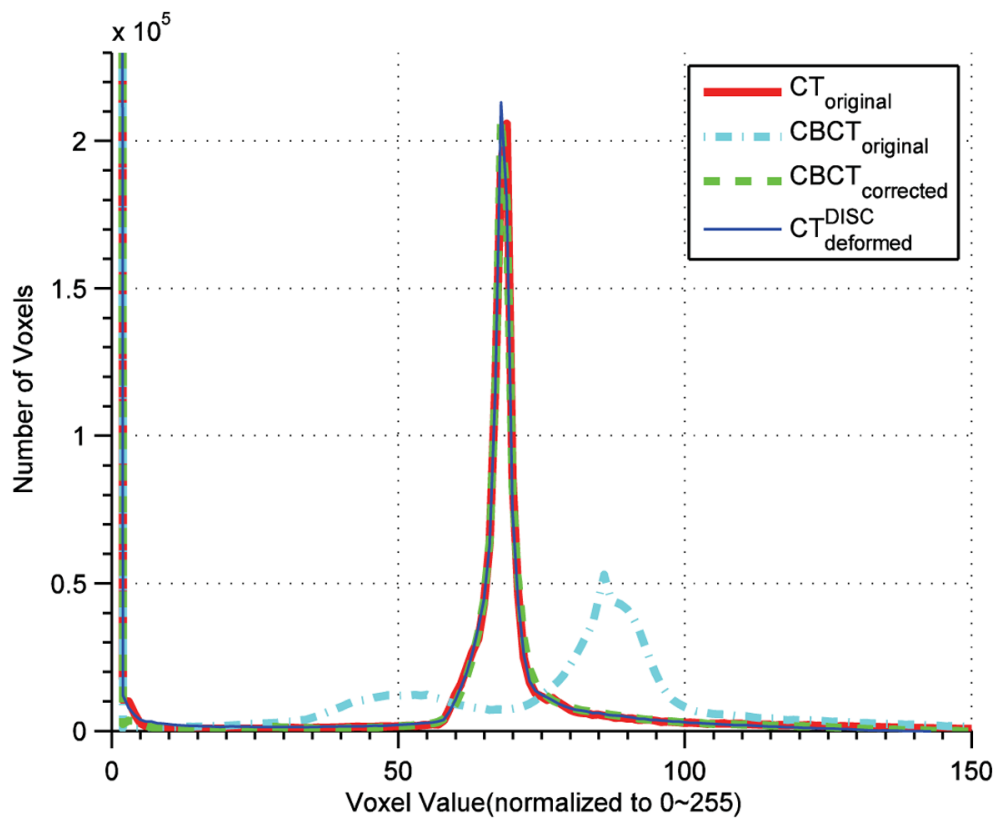

Figure 7.

Histograms of $\mathrm{CT}_{\text {original }}, \mathrm{CBCT}_{\text {original }}, \mathrm{CT}_{\text {deformed }}^{\mathrm{DISC}}$ and $\mathrm{CBCT}_{\text {corrected }}$. 

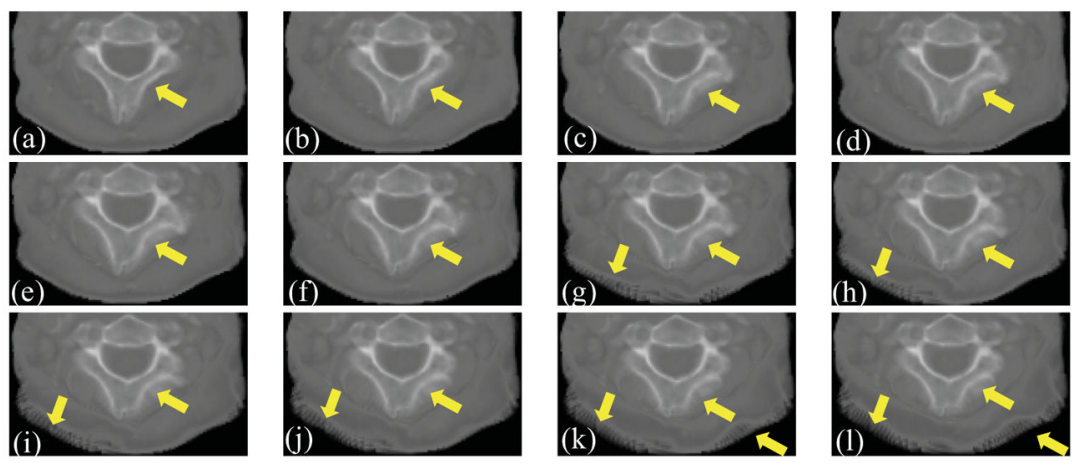

Figure 8.

Effect of patch size on the deformed CT images. (a)-(1) are part of $\mathrm{CT}_{\text {deformed }}^{\text {DISC }}$ obtained with patch size of $3 \times 3 \times 3,5 \times 5 \times 3,7 \times 7 \times 3,9 \times 9 \times 3,11 \times 11 \times 3,13 \times 13 \times 5,15 \times 15 \times 5,17 \times 17 \times 5$, $19 \times 19 \times 5,21 \times 21 \times 5,23 \times 23 \times 7,25 \times 25 \times 7$. The arrows indicate the regions of deterioration as the patch size increase. 

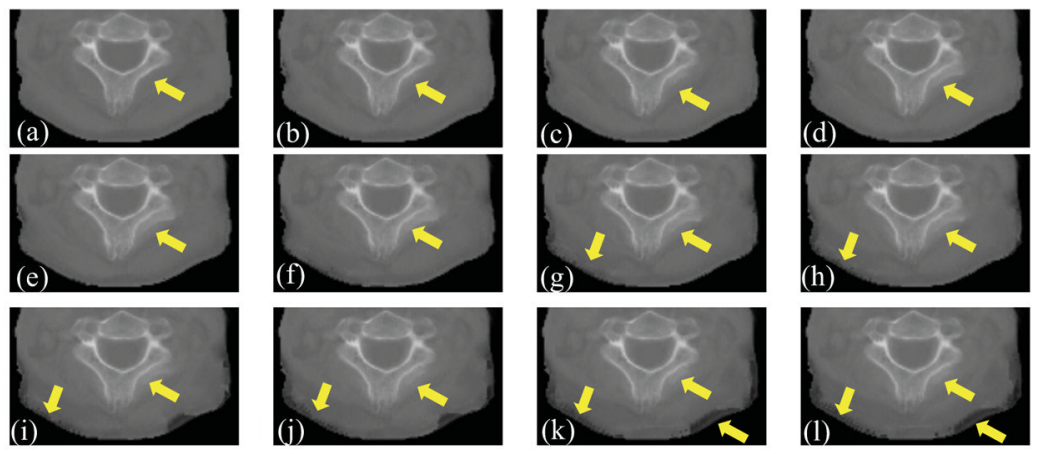

Figure 9.

Effect of patch size on the intensity corrected CBCT images. (a)-(1) are part of $\mathrm{CBCT}_{\text {corrected }}$ obtained with patch size of $3 \times 3 \times 3,5 \times 5 \times 3,7 \times 7 \times 3,9 \times 9 \times 3,11 \times 11 \times 3$, $13 \times 13 \times 5,15 \times 15 \times 5,17 \times 17 \times 5,19 \times 19 \times 5,21 \times 21 \times 5,23 \times 23 \times 7,25 \times 25 \times 7$. The arrows indicate the regions of deterioration as the patch size increase. 
(a)
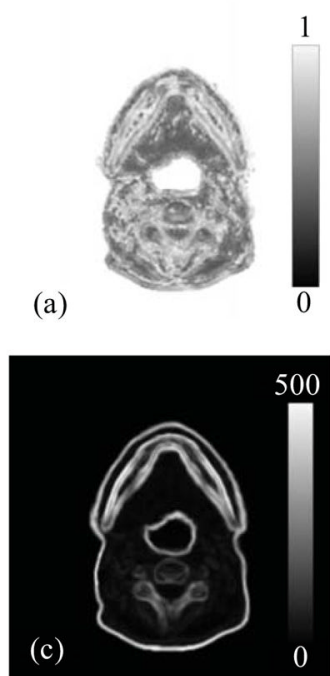

Variable a

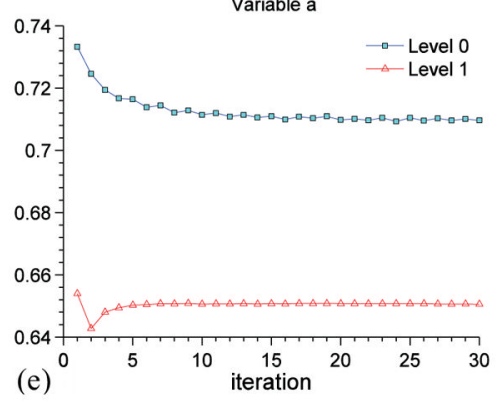

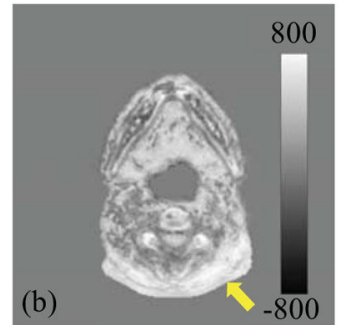
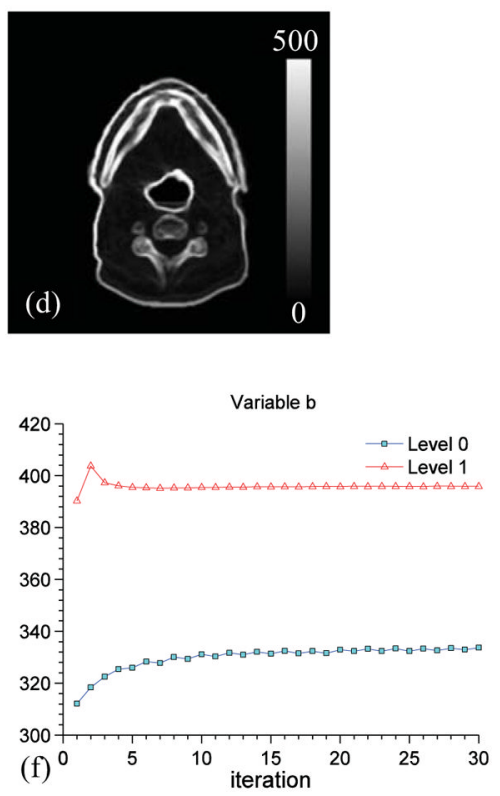

Figure 10.

(a) and (b): Distributions of parameters $a$ and $b$ on one transversal slice, respectively. The arrow indicates where the artifact presents in the original CBCT image; (c) and (d): Standard deviation of image intensities in each patch in $\boldsymbol{I}_{m}$ and $\boldsymbol{I}_{S}$ at the last iteration; (e) and (f): Evolution of average $a$ and $b$ values with iteration at two image resolution levels, respectively. The results are for Case 2 . 


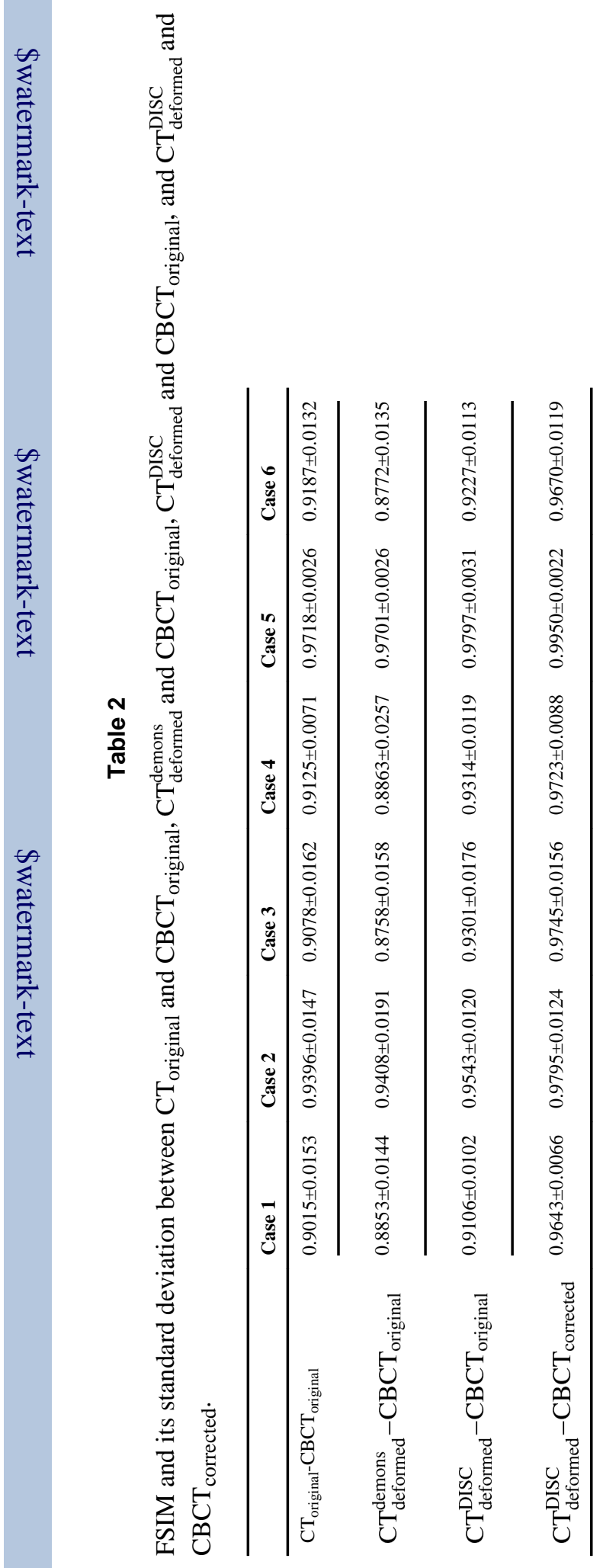




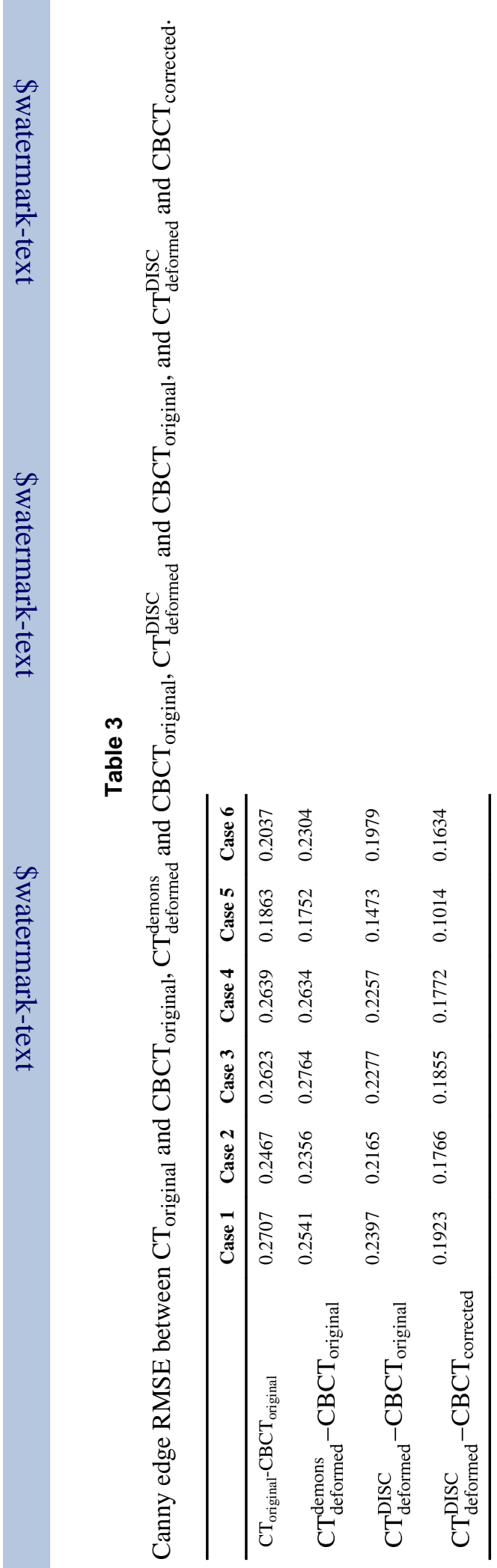


Table 4

\section{Algorithm A1}

Globally adjust the intensity of CBCT

Initialize the moving vector $\mathbf{r}$ to zero

Down-sample the images to the coarsest resolution

Repeat for each resolution level

while $1^{(k-10)}-X^{(k)} \leq \mathcal{E}$, do

1. Compute $a$ and $b$ for each voxel using patches in $\boldsymbol{I}_{m}^{(k)}$ and $\boldsymbol{I}_{s}$ (Eqs. (5), (6));

2. Interpolate/extrapolate $a$ and $b$ to where $\xi=1$;

3. Obtain $\boldsymbol{I}_{s}^{\prime}$ by applying the estimated linear transformation at each voxel;

4. Compute dr for each voxel (Eq. (1));

5. Add dr to the total moving vector $\mathbf{r}$;

6. Regularize $\mathbf{r}$ by applying a Gaussian kernel;

Up-sample the moving vector $r$ to a finer resolution level

Until the finest resolution is reached 